\title{
Epigenetic change in kidney tumor: downregulation of histone acetyltransferase MYST1 in human renal cell carcinoma
}

Yong Wang ${ }^{1,2+}$, Rui Zhang ${ }^{3,4+}$, Donglu Wu ${ }^{3,4}$, Zhihua Lu' ${ }^{1}$ Wentao Sun ${ }^{1}$, Yong Cai ${ }^{3}$, Chunxi Wang ${ }^{1 *}$ and Jingji Jin ${ }^{3^{*}}$

\begin{abstract}
Background: MYST1 (also known as hMOF), a member of the MYST family of histone acetyltransferases (HATs) as an epigenetic mark of active genes, is mainly responsible for histone H4K16 acetylation in the cells. Recent studies have shown that the abnormal gene expression of hMOF is involved in certain primary cancers. Here we examined the involvement of hMOF expression and histone H4K16 acetylation in primary renal cell carcinoma (RCC). Simultaneously, we investigated the correlation between the expression of hMOF and clear cell RCC (cCRCC) biomarker carbohydrase IX (CA9) in RCC.
\end{abstract}

Materials and methods: The frozen RCC tissues and RCC cell lines as materials, the reverse transcription polymerase chain reaction (RT-PCR), western blotting and immunohistochemical staining approaches were used.

Results: RT-PCR results indicate that hMOF gene expression levels frequently downregulated in $90.5 \%$ of patients (19/21) with RCC. The reduction of hMOF protein in both RCC tissues and RCC cell lines is tightly correlated with acetylation of histone H4K16. In addition, overexpression of CA9 was detected in 100\% of ccRCC patients (21/21). However, transient transfection of hMOF in CCRCC 786-0 cells did not affect both the gene and protein expression of CA9.

Conclusion: hMOF as an acetyltransferase of H4K16 might be involved in the pathogenesis of kidney cancer, and this epigenetic changes might be a new CA9-independent RCC diagnostic maker.

Keywords: Renal cell carcinoma, hMOF, Carbonate anhydrase IX

\section{Introduction}

Changes of chromatin structure are mainly regulated by epigenetic regulations including ATP-dependent remodeling of nucleosomes, the incorporation of variants histones into nucleosomes and posttranslational modifications of histones [1]. Post-translational modifications of the Nterminal tails of histones include acetylation, methylation, phosphorylation, ubiquitination, sumoylation, and ADPribosylation [2,3]. Histone acetylation as one of the best characterized epigenetic modifications is controlled by histone acetyltransferases (HATs) and histone deacetylases (HDAC). The balance between histone acetylation and

\footnotetext{
*Correspondence: chunxi_wang@126.com; jjin@jlu.edu.cn

${ }^{\dagger}$ Equal contributors

'Urology Department, The First Clinical Hospital, Jilin University, Changchun City, Jilin 130021, China

${ }^{3}$ College of Life Science, Jilin University, Changchun City, Jilin 130012, China Full list of author information is available at the end of the article
}

deacetylation serves as a key epigenetic mechanism for gene expression, DNA repair, developmental processes and tumorigenesis [4-6]. Thus, any reason to make this imbalance can lead to abnormal cell function, even cancer.

MYST1 (also known as hMOF), is the human ortholog of the Drosophila MOF protein containing chromodomain and acetyl-CoA binding motif which is one of the key components of the dosage compensation complex (DCC) or the male specific lethal ( $d$ MSL) complex [7]. Recent biochemical purifications revealed that hMOF forms at least two distinct multi-protein complexes in mammalian cells. One complex is the evolutionary conserved human MSL complex which is responsible for the majority of histone $\mathrm{H} 4$ acetylation at lysine $16[8,9]$. The other hMOFcontaining complex is the human non-specific lethal (NSL) complex which is recently characterized by Cai Y et al. [10]. hNSL complex can also acetylate histone $\mathrm{H} 4$ at 
lysine 5 and 8 on the recombinant polynucleosomes with the exception of histone H4K16. Although the functions of hMSL and hNSL complexes in human cells are not very clear, both complexes can acetylate histone $\mathrm{H} 4$ at lysine 16 , suggesting the importance of acetylation of H4K16 in cells. Except for acetylation of H4K16, NSL complex was found to be able to acetylate the tumor suppressor protein p53, and this acetylation is able to affect the behavior of p53 in response to DNA damage [11]. It has been reported that depletion of hMOF in human cells leads to genomic instability, spontaneous chromosomal aberrations, cell cycle defects, reduced transcription of certain genes, and defective DNA damage repair and early embryonic lethality [4-7]. This suggests a critical role for hMOF in fundamental processes such as gene transcription, cell proliferation, differentiation and DNA repair response. It is worth mentioning that depletion of hMOF also leads to global reduction of histone H4K16 acetylation in human cells $[8,12]$. However, recent studies suggest that the global modification status of H4K16Ac is also affected by Gcn-5-containing HAT and SIRT-LSD1 HDAC complexes [13,14], indicating hMOF might not be the only HAT fulfilling acetylation of H4K16 in cells. Although the role of histone H4K16 acetylation in transcription regulation is not completely understood, loss of H4K16 acetylation has been found in certain cancers. Pfister et al. [15] found that frequent downregulation of hMOF in large series of primary breast carcinomas and medulloblastomas and hMOF protein expression tightly correlated with acetylation of H4K16 in both cancers. In addition, analysis of the tissue microarray slides revealed low or absent histone H4K16 acetylation in majority of breast cancer tissues [16].

Renal cell carcinoma (RCC) is one of the most common genitourinary malignancies, accounting for about $3 \%$ of all cancers worldwide [17]. With the improved imaging diagnostic technology, more RCC cases have been diagnosed at an early stage. However, there is a considerable number of RCC patients at the time of diagnosis has been transferred [18]. Research efforts have found various biomarkers of diagnostic and prognostic of RCC such as hypoxia-induced factor 1alpha (HIF1 $\alpha)$, vascular endothelial growth factor (VEGF), and carbonic anhydrase IX (CA9), but they are not specific and sensitive enough to accurately predict the survival of RCC patients [19-21]. Recent studies indicate that epigenetic alterations play an important role in carcinogenesis, and global histone modifications as predictors of cancer recurrence in various tumor entities has begun to study. Patients with RCC have been found that total acetylation levels of histone $\mathrm{H} 3$ were inversely correlated with pTstage, distant metastasis, Fuhrman grading and RCC progression, whereas total histone H4Ac deacetylation was correlated with pT-stage and grading [22]. All the above observations strongly suggest that histone modifications might be involved in the development and progression of RCC. However, it is not clear which particular enzyme or specific modified lysine residue is responsible for tumorigenesis in RCC. This study aims to assess hMOF expression and its corresponding acetylation of histone H4K16 in the RCC via qRT-PCR, western blotting and immunohistochemistry. Simultaneously, we also investigated the correlation between the expression of hMOF and CA9.

\section{Materials and methods Materials}

Anti-H4K16 (Cat\# H9164) polyclonal antibody was purchased from Sigma. Anti-MYST1 (Cat\# A300-992A) was obtained from Bethyl Laboratories. Anti-CA9 (Cat\# sc-25599) was from Santa Cruz Biotechnology. AntiGAPDH and anti-hMOF rabbit polyclonal antibodies were raised against bacterially expressed proteins (Jilin University).

\section{Tissue collection}

Human paired clinical RCC tissues and matched adjacent tissues were collected from patients with primary RCC between March 2011 and May 2012, who underwent kidney tumor radical surgery at the First Hospital of Jilin University. The study was approved by the Ethics Committee of the First Hospital of Jilin University and all patients gave informed consent. All removed tissues during the surgery were frozen immediately in liquid nitrogen and then stored at $-80^{\circ} \mathrm{C}$. Patient medical records including tumor staging, pathological diagnosis, and surgical records were reviewed. The pathologic diagnosis of the resected tumors was based on the American Joint Committee on Cancer [23]. All patients did not receive chemotherapy or radiotherapy before surgery.

\section{Cell culture and maintenance}

Human embryonic kidney cell line HEK293T, human clear cell renal cell carcinoma (ccRCC) cell lines 786-0 (TCHu3) and MN/CA9 positive human renal cell carcinoma cell line OS-RC-2 ( $\mathrm{TCHu} 40$ ) were obtained from Cell Resource Center of Shanghai Institute of Life Science, Chinese Academy of Science. Cells were cultured in Dulbecco's Modified Eagle's Medium (DMEM, Sigma) with $5 \%$ glucose and $10 \%$ fetal bovine serum, $100 \mathrm{U} / \mathrm{mL}$ penicillin, $100 \mathrm{mg} / \mathrm{mL}$ streptomycin in $10 \mathrm{~cm}$ dishes at $37^{\circ} \mathrm{C}$ in a humidified atmosphere of $5 \% \mathrm{CO} 2$. Cultured cells were harvested from 1 well of 6-well plate and lysed using ice-cold RIPA lysis buffer (50 mM Tris $\mathrm{HCl}$ (pH7.4), $150 \mathrm{mM} \mathrm{NaCl}, 1 \%$ Nonidet P-40, $0.25 \% \mathrm{Na}$-deoxycholate, $1 \mathrm{mM}$ EDTA and protease inhibitor cocktail). Following centrifugation at 12,000 $\times \mathrm{g}$ for $15 \mathrm{~min}$ at $4^{\circ} \mathrm{C}$, total proteins in resulting supernatant was quantified using the Bradford assay following the manufacturer's instruction (BioRad). 
A

\begin{tabular}{lcccccc}
\multicolumn{6}{l}{ Table 1 Clinical information of four selected patients with ccRCC. } \\
\hline Patients & Sex & Age & $\begin{array}{c}\text { Clinical } \\
\text { diagnosis }\end{array}$ & $\begin{array}{c}\text { Nuclear } \\
\text { grade }\end{array}$ & $\begin{array}{c}\text { Tumor } \\
\text { size }(\mathrm{cm})\end{array}$ & $\begin{array}{c}\text { Tumor } \\
\text { stage }\end{array}$ \\
\hline Patient 1 & male & 34 & ccRCC & Fuhrman I & $3 \times 3 \times 2.5$ & T1a \\
Patient 2 & female & 63 & ccRCC & Fuhrman III & $6 \times 5.5 \times 4.5$ & T1b \\
Patient 3 & female & 48 & ccRCC & Fuhrman II & $4 \times 3.5 \times 2.5$ & T1b \\
Patient 4 & male & 62 & ccRCC & Fuhrman II & $6 \times 5 \times 4.5$ & T1b \\
\hline
\end{tabular}

\section{B}
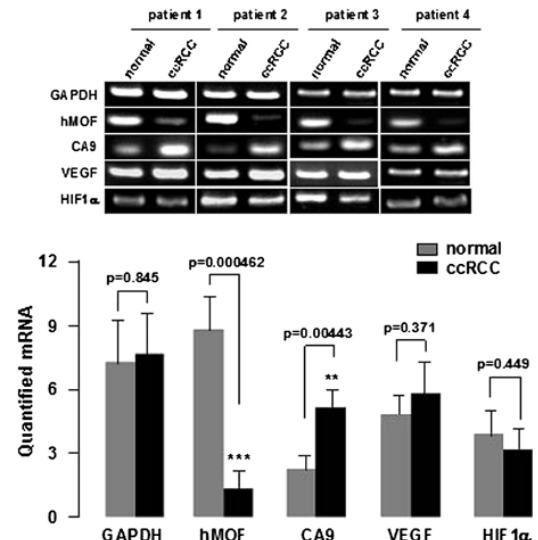

C
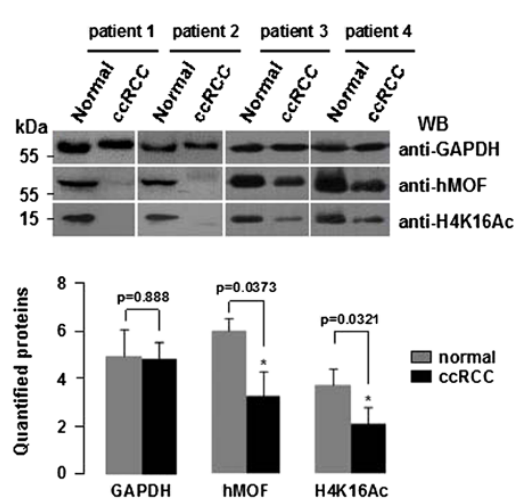

D

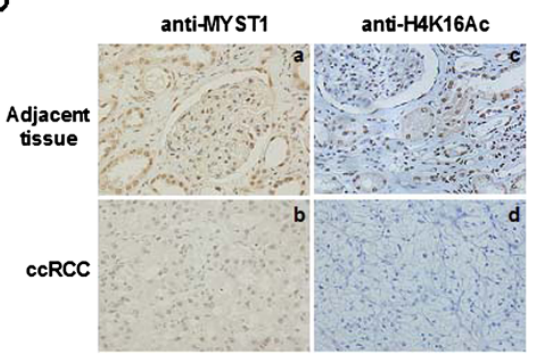

Figure 1 hMOF is downregulated in human ccRCC. A. Clinical informations of four newly diagnosed patients with ccRCC. B. hMOF mRNA levels are dropped down in 4 random cases of ccRCC tissues. Total RNA from tissue was isolated using trizol. mRNA levels of hMOF, CA9, VEGF and HIF1a in paired human clinical cCRCC and adjacent kidney tissue was analyzed by RT-PCR (upper panel). mRNA levels were quantified by densitometry using Quantity One Basic software (Bio- Rad) (lower panel). C. Total hMOF protein expression and the acetylation of histone H4K16 levels are decreased in selected ccRCC tumor tissue. Aliquots of whole cell extracts from four selected ccRCC tumor samples and its corresponding adjacent tissues were subjected to SDS-PAGE in 12\% gels, and proteins were detected by western blotting with indicated antibodies (upper panel). Western blot images were quantified using Quantity One software (Bio-Rad) (lower panel). The significant difference is expressed as ${ }^{*} p<0.05,{ }^{* *} p<0.01,{ }^{* *} p<0.001$. D. An example of immunostaining for hMOF and H4K16Ac in ccRCC. hMOF expression status in adjacent renal tissue (a) and in CCRCC (b) were visualized by immunohistochemical staning with anti-MYST1 antibody. Acetylation levels of modified histone H4K16 was immunostained by acetylation-specific antibody in adjacent renal tissue (c) and in ccRCC (d).

\section{Western blotting}

Aliquot of whole cell extract from cultured cells was mixed with $4 x$ SDS sample buffer $(0.25 \mathrm{M}$ Tris- $\mathrm{HCl}$ pH $6.8,8 \%$ SDS, 30\% Glycerol, $0.02 \%$ Bromophenol Blue containing $10 \%$ BME). Denatured proteins were separated by SDS polyacrylamide gel (SDS-PAGE) and specific proteins were analyzed by western blotting. $200 \mathrm{mg}$ of kidney tissue samples were homogenized with liquid nitrogen and solubilized in $200 \mu \mathrm{l}$ cold PBS containing 1.0\% Nonidet P-40, 0.5\% Na-deoxycholate, 0.1\% SDS, $0.05 \mathrm{mM}$ PMSF and protease inhibitor cocktail. The homogenate was swirled and kept on ice for 30 minutes. Whole cell extracts were prepared by sonication (SCIENTZ-IID, China) for $10 \mathrm{sec}-$ onds with $50 \%$ duty cycle and centrifugation at $12,000 \mathrm{rpm}$ for $15 \mathrm{~min}$. Spectrophotometer used to measure protein concentrations in a solution using a Bradford assay kit. Equal total amounts of denatured proteins were separated by SDS-PAGE. Specific proteins were detected by immunoblotting using hMOF, H4K16Ac, CA9 and GAPDH polyclonal antibodies. Immunoblotted proteins were visualized using the chemiluminescent detection system (PierceTechnology).

\section{Reverse transcription PCR (RT-PCR)}

Cells were harvested from 1 well of a 6-well plate and total RNA was isolated using TRIzol ${ }^{\circledR}$ LS Reagent (Invitrogen). Total RNA from kidney tissues (normal/adjacent or tumor) were also isolated using TRIzol ${ }^{\circledR}$ LS Reagent. $1 \mu \mathrm{g}$ of RNA from each sample was used as a template to produce cDNA with PrimeScript 1st Strand cDNA Synthesis Kit (TAKARA). hMOF, CA9 and GAPDH mRNA levels were analyzed by Polymerase chain reaction (PCR) with $\mathrm{C} 1000^{\mathrm{TM}}$ Thermal Cycler (BIO-RAD) and quantitative real time PCR with Real Time PCR Detector Chromo 4 (BIORAD). All PCR reactions were finished under following program: initial denaturation step was $95^{\circ} \mathrm{C}$ for $3 \mathrm{~min}$, followed by 35 cycles of denaturation at $95^{\circ} \mathrm{C}$ for 30 seconds, annealing at $60^{\circ} \mathrm{C}$ for 30 seconds and extension at $72^{\circ} \mathrm{C}$ for 30 seconds. The primer sets used for PCR were as follows: GAPDH, 5'-ATCACTGCCACCCAGAAGAC-3' (forward) and 5'-ATGAGGTCCACCACCCTGTT-3' (reverse), yielding a 460 bp product; CA9, 5'-GCAGGAG GATTCCCCCTTG-3 (Forward) and 5'-GGAGCCTC AACAGTAGGTAGAT-3' (Reverse), yielding a 185 bp product; hMOF, 5'-GGCTGGACGAGTGGGTAGACAA- 


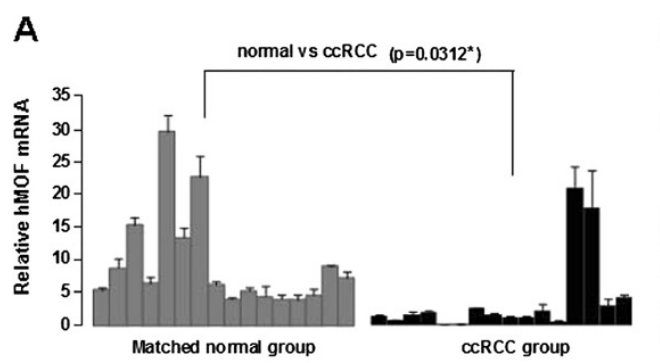

B

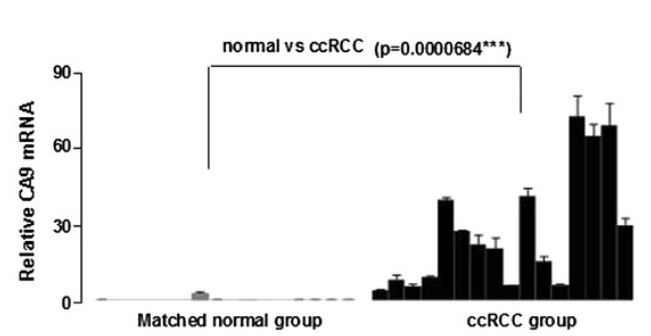

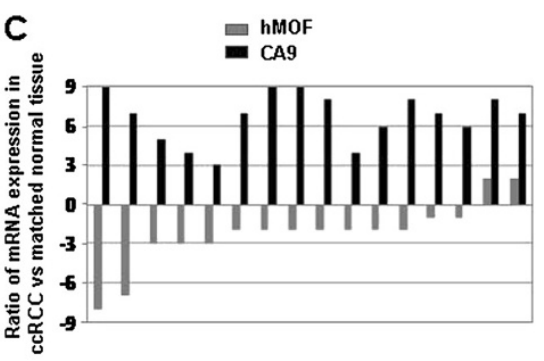

D

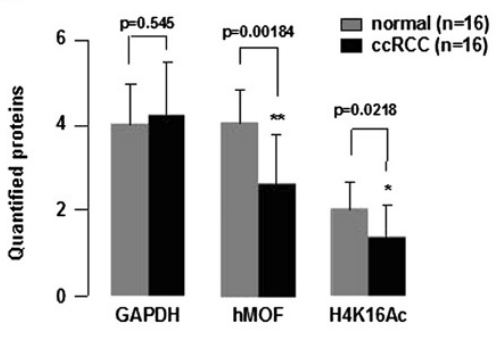

Figure 2 Downregulation of hMOF is accompanied by increased CA9 in ccRCC. A-B. Relative mRNA expression levels of hMOF and CA9 in cCRCC. Total RNA was isolated from sixteen paired clinical ccRCC and adjacent kidney tissues. Relative mRNA expression levels of hMOF and CA9 were analized by quantitative RT-PCR. Error bars represent the standard error of the mean of 3 independent experiments. Student's $t$-test was performed to compare the difference between cCRCC and normal tissues. C. Expression patterns of hMOF and CA9 mRNAs in cCRCC and its corresponding adjacent kidney tissues. Expression is displayed as a ratio of expression of hMOF or CA9 gene in ccRCC versus matched normal tissues. Each bar is the log2 value of the ratio of hMOF or CA9 expression levels between ccRCC and matched normal tissues from the same patients. Bar value $>1$ represents $>2$-fold increased, whereas bar value $<-1$, represents $>2$-fold decreased. D. Protein expression levels of hMOF and its H4K16Ac status in cCRCC. Aliquots of whole cell extracts from sixteen selected ccRCC tumor samples and its corresponding adjacent tissues were analyzed by western blotting. The blots were then scanned and quantified with Quantity One software. The significant difference is expressed as ${ }^{*} p<0.05,{ }^{* *} p<0.01$.

$3^{\prime}$ (forward) and 5'-TGGTGATCGCCTCATGCTCCTT-3' (reverse), yielding a 227 bp product.

\section{Immunohistochemical staining}

Formalin-fixed and paraffin-embedded clear cell renal carcinoma tissue blocks were from the The First Clinical Hospital of Jilin University. Tissue blocks were sectioned and deparaffinized in xylene and rehydrated through a graded ethanol series. Tissue slides were then subjected to antigen retrieval by boiling in $0.01 \mathrm{M}$ sodium citrate buffer (pH 6) in a microwave oven for $10 \mathrm{~min}$. Endogenous peroxidase was blocked by incubation for $10 \mathrm{~min}$ in $3 \%$ hydrogen peroxide in methanol. Finally, the reactions were detected using the DAB detection kit (Dako). AntiMYST1 and acetylated H4K16 polyclonal antibodies were used at a 1:500 dilution. MYST1 protein expression status and the histone H4K16 acetylation levels were estimated in a four-step scale (none, weak, moderate, strong). The determination criteria are shown below: score $0=$ none, no staining or nuclear staining $<10 \%$ of tumor cells; score 1 = weak, partial or weak complete nuclear staining $>10 \%$ of tumor cells; score 2 = moderate complete nuclear staining $>10 \%$ of tumor cells; score $3=$ strong and complete nuclear staining in $>10 \%$ of tumor cells [24].

\section{Transient transfection}

Human embryonic kidney (HEK) 293T cells, renal cell carcinomas 786-0 and OS-RC-2 cells were cultured in 6 well tissue culture plates $\left(\sim 2 \times 10^{5}\right.$ cells/well $)$ in DMEM containing $10 \%$ fetal bovine serum and antibiotics. The cells were transiently transfected with $0.25 \sim 2 \mu \mathrm{g}$ of hMOF cDNAs using polyethylenimine (PEI). At $48 \mathrm{hrs}$ post-transfection, cells were harvested and lysed for immunoblot and RT-PCR analysis.

\section{Statistical analysis}

The expression difference of genes and proteins between ccRCC and normal tissues were statistically analyzed. Statistical analysis was completed with SPSS 17.0 (SPSS, Inc., Chicago IL). Statistical comparisons were analyzed using the student's $t$-test. Values of $P<0.05$ were considered to be statistically significant.

\section{Results}

Downregulation of hMOF mRNA in primary renal cell carcinoma tissues

In order to know whether the hMOF is involved in the pathogenesis of primary RCC or not, we first examined the mRNA levels of hMOF and other hypoxia signature genes including CA9, VEGF and HIF1 $\alpha$ in 4 random 

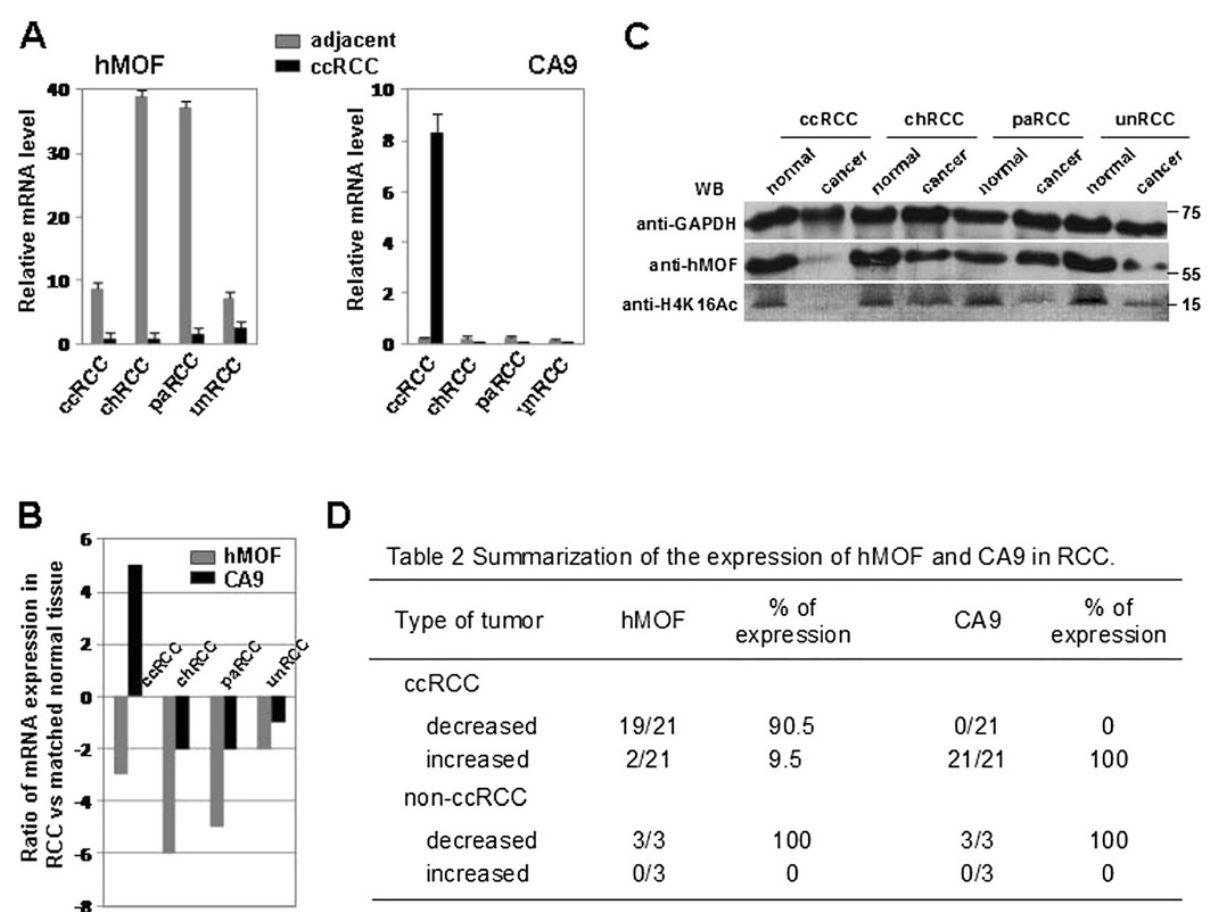

D

\begin{tabular}{ccccc}
\multicolumn{4}{c}{ Table 2 Summarization of the expression of hMOF and CA9 in RCC. } \\
\hline Type of tumor & hMOF & $\begin{array}{c}\% \text { of } \\
\text { expression }\end{array}$ & CA9 & $\begin{array}{c}\% \text { of } \\
\text { expression }\end{array}$ \\
\hline ccRCC & & & \\
decreased & $19 / 21$ & 90.5 & $0 / 21$ & 0 \\
increased & $2 / 21$ & 9.5 & $21 / 21$ & 100 \\
non-ccRCC & & & & \\
decreased & $3 / 3$ & 100 & $3 / 3$ & 100 \\
increased & $0 / 3$ & 0 & $0 / 3$ & 0 \\
\hline
\end{tabular}

Figure $3 \mathrm{hMOF}$ is downregulated in different pathological diagnosis of human kidney cancer. A. Relative mRNA expression levels of hMOF in different type of kidney cancer. Total RNA was isolated from four paired pathological diagnosed cCRCC, chRCC, paRCC, unclassified RCC, respectively and matched normal/adjacent kidney tissues. Relative mRNA expression levels of hMOF and CA9 were analyzed by qRT-PCR. Error bars represent the standard error of the mean of 3 independent experiments. B. Log2 ratio of hMOF and CA9 mRNA expression in four different types of human kidney cancer. Ratio of mRNA expression is displayed as a ratio of expression of hMOF or CA9 gene in ccRCC versus matched normal tissues. C. Analysis of werstern blotting. Equivalent total protein amount of whole cell extracts from four different pathological diagnosed kidney cancers (cCRCC, chRCC, paRCC and unRCC) and its corresponding normal/adjacent tissues were subjected to SDS-PAGE in 12\% gels, and proteins were detected by western blotting with indicated antibodies. D. Summarization of hMOF and CA9 expression in RCC. Total cases of cCRCC (21) include four initial selected ccRCC (data not shown), sixteen additional ccRCC and one case used in comparing experiment.

cases of newly diagnosed ccRCC (Figure 1A) by reverse transcription PCR (RT-PCR) and quantitative real-time PCR (qPCR). As shown in Figure 1B, the gene expression levels of hMOF were markedly decreased in all ccRCC tissues compared to matched normal tissues $(p<0.001)$. In contrast, CA9 expression levels were significantly increased in all ccRCC tissues $(\mathrm{p}<0.01)$. However, no significant difference was observed in VEGF and HIF1 $\alpha$ expression. Additional 16 paired clinical ccRCC and matched normal tissues were used to further validate the frequent downregulation of hMOF mRNA expression in primary ccRCC. Analysis of performed mRNA expression of 16 samples revealed significant ( $>2$-fold decreased) downregulation of hMOF mRNA in $87.5 \%(14 / 16)$ of patients (Figure $2 \mathrm{~A}$ and $\mathrm{C}$ ), whereas $12.5 \%(2 / 16)$ of patients showed significant (>2-fold increased) upregulation of hMOF (Figure 2A and C). However, less relationship between hMOF expression and tumor size, stage and grading was detected in our limited number of cases (data not shown). To examine the gene expression status of hMOF in other types of
RCC, four kidney cancer patients with pathologically daignosed ccRCC, chRCC (chromophobe RCC), paRCC (papillary RCC) and unRCC (unclassified RCC), respectively, were selected. Analysis of qRT-PCR results showed that the gene expression of hMOF significantly downregulated in all types of RCC ( $>2$-fold) (Figure 3A and B).

\section{Reduction of hMOF protein in human primary renal cell carcinoma tissues}

The results of RT-PCR analysis clearly show frequent downregulation of hMOF gene expression in RCC. To determine whether the reduction of hMOF mRNA expression resulted in decreasing of hMOF protein levels, western blotting and immunohistochemical staining approaches were used. As shown in Figure 1C, aliquots of whole cell extract from four paired initially selected ccRCC and matched normal tissues were analyzed by western blotting with indicated antibodies. Similar to our expected results, significant reduction of hMOF protein in ccRCC compared to those of matched normal tissues were detected $(\mathrm{p}<0.05)$. Simultaneously, the 
A

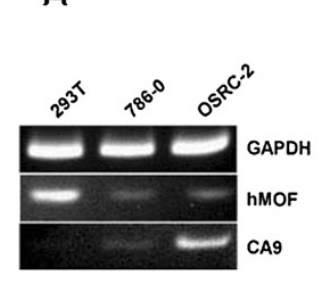

C

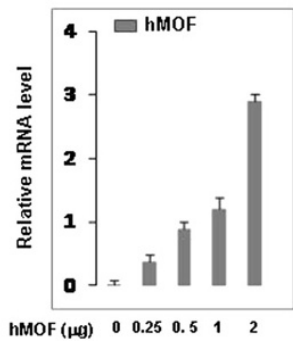

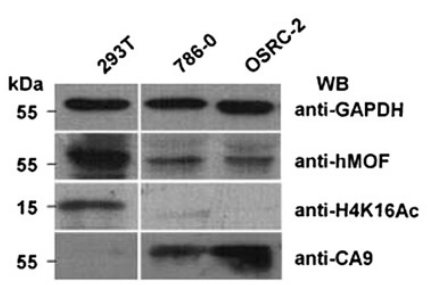

D

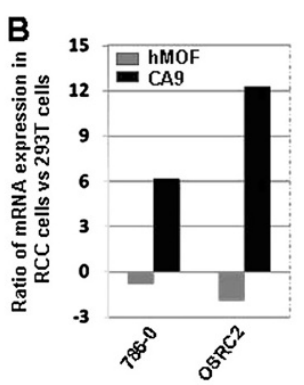

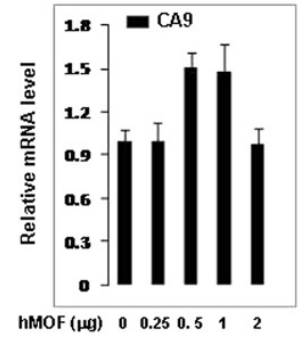

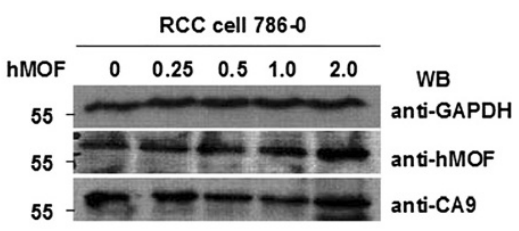

Figure 4 Non-correlation between hMOF and CA9 is found in renal cell carcinoma cells. A. hMOF protein expression was correlated with acetylation of H4K16 in RCC cell 786-0 and OSRC-2. 293T, 786-0 or OSRC-2 cells were cultured in 6-well tissue culture plates ( 2x10 cells/well) in DMEM medium containing 10\% fetal bovine serum. Whole cell extracts were subjected to immunoblotting using indicated antibodies (right panel). 293T, 786-0 or OSRC-2 cells from 1 well of a 6 well plate were lysed and total RNA was isolated using Trizol. hMOF and CA9 gene expressions were measured by RT-PCR (left panel) and qRT-PCR (B). C. Effect of hMOF on CA9 mRNA expression levels in RCC cells. RCC 786-0 cells were cultured in 6-well tissue culture plates $\left(\sim 2 \times 10^{5}\right.$ cells/well) in DMEM medium containing $10 \%$ fetal bovine serum. The cells were transfected with $0.25,0.5,1$ and $2 \mu \mathrm{g}$ of hMOF cDNAs. 48 hours after transfection, cells were lysed and total RNA was isolated using Trizol. Indicated gene expressions were analyzed by qRT-PCR. D. Effect of hMOF on CA9 protein expression in RCC cells. RCC 786-0 cells were transfected with $0.25,0.5,1$ and $2 \mu \mathrm{g}$ of hMOF cDNAs. 48 hours after transfection, cells were harvested and lysed in RIPA buffer. Aliquots of whole cell extracts were subjected to $12 \%$ SDS-PAGE, and specific proteins were detected by indicated antibodies.

acetylation status of histone H4K16 was also significantly reduced or lost $(\mathrm{p}<0.05)$. To further confirm these results, we performed immunohistochemical staining for hMOF and histone H4K16 acetylation in the formalin fixed paraffin embedded tissue sections of same four selected ccRCC patients. The results revealed that both the hMOF protein levels and the histone H4K16 acetylation status were markedly reduced (score 1 to 2 for hMOF staining, and score $0-1$ for H4K16Ac staining) in all ccRCC tissues compared to adjacent tissues. For example, the results of immunohistochemical staining for hMOF and H4K16Ac are presented in Figure 1D. Weak staining of hMOF and no staining of H4K16Ac in the ccRCC paraffin embedded tissue sections were detected. In the additional 16 paired clinical ccRCC and matched normal tissues, hMOF protein expression and H4K16Ac status were detected by western blotting. The quantified protein levels (Quantity One software) were analyzed by $t$-test. As shown in Figure 2D, hMOF protein expression levels were significantly reduced in ccRCC tissues $(\mathrm{p}<0.01)$, and the expression of hMOF was tightly correlated with $\mathrm{H} 4 \mathrm{~K} 16$ acetylation $(\mathrm{p}<0.05)$. Furthermore, in the four different pathologically diagnosed ccRCC, chRCC, paRCC and unRCC, hMOF protein expression was significantly decreased in ccRCC, chRCC and unclassified RCC, whereas less changes were detected in paRCC (Figure 3C).

Elevation of CA9 gene expression is accompanied by frequent reduction of hMOF MRNA in ccRCC

CA9 is not expressed in healthy renal tissue but is expressed in most ccRCC through HIF1 $\alpha$ accumulation driven by hypoxia [25]. In our study, the gene expression of CA9 was significantly increased ( $>2$-fold) in $100 \%$ of ccRCC patients (21/21; Figure 3D) including four initial selected ccRCC, sixteen additional ccRCC (Figure 2B) and one case (Figure $3 \mathrm{~A}$ and $\mathrm{B}$ ) used in comparing experiment. Among these cases, reduction of hMOF mRNA expression was detected in $90.5 \%$ of cases $(19 / 21)$. There were only 2 cases presenting elevation of hMOF mRNA expression in ccRCC (Figure 2A and C). However, no elevation of CA9 gene expression was detected in different pathologically diagnosed RCC including chRCC, paRCC and unRCC, although the mRNA levels of hMOF were significantly decreased in those RCC (Figure 3B).

\section{Non-correlation between hMOF and CA9 is found in renal cell carcinoma cells}

To extend these observations and to know whether there is a correlation regulatory relationship between 
hMOF and CA9 in cells, we performed experiments using RCC cell 786-0 and OS-RC-2 as model. We first examined both the protein levels and mRNA expression levels of the hMOF and CA9 in 293T, 786-0 and OS$\mathrm{RC}-2$ cells. The results as shown in Figure 4A indicate the opposing gene expression patterns between hMOF and CA9 were observed. The expression of hMOF was reduced in both 786-0 and OS-RC-2 cells compared to $293 \mathrm{~T}$ cells, and the $\log 2$ ratio changes are -0.84 and -1.9 , respectively. Western blotting analysis revealed that the hMOF proteins were markedly decreased in both renal cell carcinoma cells. In addition, the reduction of hMOF proteins resulted in loss of the acetylation of histone H4K16 in RCC cells. In contrast with hMOF, the gene expression of CA9 was increased in both $786-0(\log 2=6.2)$ and OS-RC-2 cells $(\log 2=12.3)$ compared to $293 \mathrm{~T}$ cells. To determine whether the CA9 gene expression was regulated by hMOF, renal cell carcinoma 786-0 cells were transiently transfected with 0.25 to $2 \mu \mathrm{g}$ of hMOF cDNAs. The results are shown in Figure $4 \mathrm{C}$ and D, both the gene and protein expression levels of hMOF were dosedependently increased. However, neither the gene nor protein expression of CA9 levels were affected by transient transfection RCC 786-0 cells with hMOF cDNAs.

\section{Discussion}

The HAT hMOF belongs to the MYST (Moz-Ybf2/Sas3Sas2-Tip60) family, and is believed to be responsible for histone $\mathrm{H} 4$ acetylation at lysine 16 in both Drosophila and human cells $[7,8,12]$. Abnormal expression of the hMOF and its corresponding modification of H4K16 have been found in certain primary cancer tissues. The expression behavior of hMOF in different primary cancers was observed to be different. Frequent downregulation of hMOF expression was found in primary breast cancer and medulloblastoma [15]. On the contrary, hMOF was overexpressed in non-small cell lung carcinoma tissues [26]. Regardless of what type of situation, hMOF protein expression tightly correlated with acetylation of histone H4K16. In this study, we investigated the expression of histone acetyltransferase hMOF and its corresponding H4K16 acetylation in a series of primary kidney tumor tissues by qRT-PCR, western blotting, and immunohistochemistry. The results revealed that either hMOF mRNA expression or hMOF protein expression was frequently downregulated in human RCC (19/21 cases; $>90 \%$ ), and hMOF protein expression was correlated with acetylation of histone H4K16 in parallel. In addition, low protein expression levels of hMOF and loss of histone H4K16 acetylation were detected in renal carcinoma cells 786-0 and OS-RC-2 compared to human embryonic kidney cell HEK293T. Together this, HAT hMOF might have an important role in primary renal cell carcinoma tumorigenesis.
CA9 is a transmembrane, zinc-containing metalloenzyme that catalyzes reversible reactions of the bicarbonate buffer system to regulate $\mathrm{pH}$ in hypoxic conditions [27]. Overexpression of CA9 has been shown in a wide variety of malignant cell lines and tumors [28-30]. It is worth mentioning that CA9 has been well described as a diagnostic marker for clear cell renal carcinoma (ccRCC), especially by showing high expression in metastastic ccRCC (mccRCC) [31,32]. Therefore, the inhibitor or regulatory proteins of hypoxic tumor-associated CA9 possesses the potential therapeutic possibility for those tumors in which CA9 is involved in perturbing the extra- or intra- tumoral acidification process. In our experiments, although the expression of VEGF and HIF1 $\alpha$ which are hypoxia signature genes were not observed significant difference between ccRCC and normal tissues, overexpression of CA9 was observed in $100 \%$ of ccRCC cases and in both renal carcinoma cell lines. Interestingly, in four different diagnostic RCCs, downregulation of hMOF was detected in all types of RCCs, but the overexpression of CA9 was only presented in ccRCC, suggesting that hMOF might be a new common diagnostic marker for human different diagnostic RCC. Although frequent downregulation of hMOF and overexpression of CA9 were detected in both RCC clinical tissues and RCC cell lines, non-correlation between hMOF and CA9 was found in RCC 786-0 cells, suggesting hMOF and its corresponding modifications might be a new CA9-independent RCC diagnosis biomarker. Although large series of clinical cases and analyses of overall survival need to be investigated, the molecular mechanism linking loss of hMOF expression to renal cell carcinoma, especially mechanism of hMOF on renal cell carcinomas, will be an exciting avenue for further research.

\section{Conclusion}

In conclusion, hMOF as an acetyltransferase of H4K16 might be involved in the pathogenesis of renal cell carcinoma, and this epigenetic change might be a new CA9-independent RCC diagnostic marker. In addition, our results suggest that a novel molecular mechanism of hMOF might serve as a lead to new therapeutics target in human renal cell carcinoma.

\section{Competing interests}

The authors declare that they have no competing interests.

\section{Authors' contributions}

YW, RZ, DW and ZL carried out the experiments and data analyses. WS and CW collected the clinical samples and completed immunohistochemistry. YC and $J$ J drafted the manuscript. All authors read and approved the final manuscript.

\section{Acknowledgements}

This work was supported by National Natural Science Foundation of China (No. 31070668, JJ) and Research Fund for the Doctoral Program of Higher Education of China (No. 20110061110020, JJ). 


\section{Author details}

'Urology Department, The First Clinical Hospital, Jilin University, Changchun City, Jilin 130021, China. 'Urology Department, Jilin province People's Hospital, Changchun City, Jilin 130021, China. ${ }^{3}$ College of Life Science, Jilin University, Changchun City, Jilin 130012, China. ${ }^{4}$ Graduate School of Jilin University, Changchun City, Jilin 130012, China.

Received: 11 December 2012 Accepted: 2 February 2013

Published: 9 February 2013

\section{References}

1. Jin J, Cai Y, Li B, Conaway RC, Workman JL, Conaway JW, Kusch T: In and out: histone variant exchange in chromatin. Trends Biochem Sci 2005, 30:680-687.

2. Berger SL: The complex languige of chromatin regulation during transcription. Nature 2007, 447:407-412.

3. Bhaumik SR, Smith E, Shilatifard A: Covalent modifications of histones during development and disease pathogenesis. Nat Struct Mol Biol 2007, 14:1008-1016.

4. Carrouzza MJ, Utley RT, Workman JL, Cote J: The divers functions of histone acetyltransferase complexes. Trends Genet 2003, 19:321-329.

5. Gupta A, Guerin-Peyrou TG, Sharma GG, Park C, Agarwal M, Ganju RK, Pandita S, Choi K, Sukumar S, Pandita RK, Ludwig T, Pandita TK: The mammalian ortholog of Drosophla MOF that acetylates histone $\mathrm{H} 4$ lysine 16 is essential for embryogenesis and oncogenesis. Mol Cell Biol 2008, 28:397-409.

6. Sharma GG, So S, Gupta A, Kumar R, Cayrou C, Avvakumov N, Bhadra U, Pandita RK, Porteus MH, Chen DJ, Cote J, Pandita TK: MOF and histone H4 acetylation at lysine 16 are critical for DNA damage response and double-strand break repair. Mol Cell Biol 2010, 30:3582-3595.

7. Rea S, Xouri G, Akhtar A: Males absent on the first (MOF): from flies to humans. Oncogene 2007, 26:5385-5394.

8. Smith ER, Cayrou C, Huang R, Lane WS, Côtê J, Lucchesi JC: A human protein complex homologus to the Drosophila MSL complex is responsible for the majority of histone $\mathrm{H} 4$ acetylation at lysine 16 . Mol Cell Biol 2005, 25:9175-9188.

9. Mendjan S, Taipale M, Kind J, Holz H, Gebhardt P, Schelder M, Vermeulen M, Buscaino A, Duncan K, Mueller J, Wilm M, Stunnenberg HG, Saumweber H, Akhtar A: Nuclear pore components are involved in the transcriptional regulation of dosage compensation in Drosophila. Mol Cell 2006, 21:811-823.

10. Cai Y, Jin J, Swanson SK, Cole MD, Choi SH, Florens L, Washburn MP, Conaway JW, Conaway RC: Subunit composition and substrate specificity of a MOF-containing histone acetyltransferase distinct from the malespecific lethal (MSL) complex. J Biol Chem 2010, 285:4268-4272.

11. Sykes SM, Mellert HS, Holbert MA, Li K, Marmorstein R, Lane WS, McMahon SB: Acetylation of the p53 DNA-binding domain regulates apoptosis induction. Mol Cell 2006, 24:841-851.

12. Taiple M, Rea S, Richter K, Vilar A, Lichter P, Imhof A, Akhtar A: hMOF histone acetyltransferase is required for histone $\mathrm{H} 4$ lysine 16 acetylation in mammalian cells. Mol Cell Biol 2005, 25:6798-6810.

13. Mulligan P, Yang F, Di Stefano L, Ji JY, Ouyang J, Nishikawa JL, Toiber D, Kulkarni M, Wang Q, Najafi-Shoushtari SH, Mostoslavsky R, Gygi SP, Gill G, Dyson NJ, Näär AM: A SIRT-LSD1 Co-repressor complex regulates notch target gene expression and development. Mol Cell 2011, 42:689-699.

14. Orpinell M, Fournier M, Riss A, Nagy Z, Krebs AR, Frontini M, Tora L: The ATAC acetyl transferase complex controls mitotic progression by targeting non-histone substrates. EMBO J 2010, 29:2381-2394.

15. Pfister S, Rea S, Taipale M, Mendrzyk F, Straub B, Ittrich C, Thuerigen O, Sinn HP, Akhtar A, Lichter P: The histone acetyltransferase hMOF is frequently downregulated in primary breast carcinoma and medulloblastoma and constitutes a biomarker for clinical outcome in medulloblastoma. Int $J$ Cancer 2008, 122:1207-1213.

16. Elsheikh S, Green AR, Rakha EA, Powe DG, Ahmed RA, Collins HM, Soria D, Garibaldi JM, Paish CE, Ammar AA, Grainge MJ, Ball GR, Abdelghany MK, Martinez-Pomares L, Heery DM, Ellis IO: Globle histone modifications in breast cancer correlate with tumor phenotypes, prognostic factors, and patient outcome. Cancer Res 2009, 69:3802-3809.

17. Jemal A, Siegel E, Ward E, Murray $T, X u$ J, Thun MJ: Cancer stastistics. CA Cancer J Clin 2007, 57:43-66.

18. Janzen NK, Kim HL, Figlin RA, Belldegrun AS: Surveillance after radical or partial nephrectomy for localized renal cell carcinoma and management of recruitment disease. Urol Clin North Am 2003, 30:843-852.
19. Eichelberg $C$, Junker $\mathrm{K}$, Ljungberg $\mathrm{B}$, Moch $\mathrm{H}$ : Diagnostic and prognostic molecular markers for renal cell carcinoma: a critical appraisal of the current state of research and clinical applicability. Eur Urol 2009, 55:851-863.

20. Belldegrun AS: Renal cell carcinoma: prognostic factors and patient selection. Eur Urol Suppl 2007, 6:477-483.

21. Wu XR, Sha JJ, Liu DM, Chen YH, Yang GL, Zhang J, Xhen YY, Bo J, Huang YR: High expression of p53-induced ring-h2 protein is associated with poor prognosis in clear cell renal cell carcinoma. Eur J Sur Oncol 2012, 39:100-106.

22. Mosashvilli D, Kahl P, Mertens C, Holzapfel S, Rogenhofer S, Hauser S, Büttner R, Von Ruecker A, Müller SC, Ellinger J: Globle histone acetylation levels: prognostic relevance in patients with renal cell carcinoma. Cancer Sci 2010, 101:2664-2669.

23. Edge SB, Byrd DR, Compton CC, Fritz AG, Greene FL, Trotti A: AJCC Cancer Staging Manual. 7th edition. Chicago, IL: Springer; 2010

24. Choschzick M, Oosterwijl R, Muller V, Woelber $L$, Simon R, Moch $H_{1}$ Tennstedt P: Overexpression of carbonic anhydrase IX (CAIX) is an independent unfavorable prognostic marker in endometrioid ovarian cancer. Virchows Arch 2011, 459:193-200.

25. Tostain J, Li G, Gentil-Perret A, Gigante M: Carbonate anhydrase 9 in clear cell renal cell carcinoma: A marker for diagnosis, prognosis and treatment. Eur J Cancer 2010, 46:3141-3148.

26. Song JS, Chun SM, Lee JY, Kim DK, Kim YH, Jang SJ: The histone acetyltransferase hMOF is overexpressed in non-small cell lung carcinoma. Korean J Pathol 2011, 45:386-396.

27. Stillebroer AB, Mulders PF, Boerman OC, Oyen WJ, Oosterwijk E: Carbonic anhydrase IX in renal cell carcinoma: implications for prognosis, daignosis, and therapy. Eur Urol 2010, 58:75-83.

28. Hussain SA, Ganesan R, Reynolds G, Gross L, Stevens A, Pastorek J, Murray PG, Perunovic B, Anwar MS, Billingham L, James ND, Spooner D, Poole CJ, Rea DW, Palmer DH: Hypoxia-regulated carbonic anhydrase IX expression is associated with poor survival in patients with invasive breast cancer. Br J Cancer 2007, 96:104-109.

29. Klatte T, Seligson DB, Rao JY, Yu H, de Martino M, Kawaoka K, Wong SG, Belldegrun AS, Pantuck AJ: Carbonic anhydrase IX in bladder cancer: a diagnostic, prognostic, and therapeutic molecular marker. Cancer 2009, 115:1448-1458.

30. Swinson DE, Jones JL, Richardson D, Wykoff C, Turley H, Pastorek J, Harris $\mathrm{AL}, \mathrm{O}$ 'Byrne $\mathrm{KJ}$ : Carbonic anhydrase IX expression, a novel surrogate marker of tumor hypoxia, is associated with a poor prognosis in nonsmall-cell lung cancer. J Clin Oncol 2003, 21:473-482.

31. Leibovich BC, Sheinin Y, Lohse CM, Thompson RH, Cheville JC, Zavada J, Kwon ED: Carbonic anhydrase IX is not an independent predictor of outcome for patients with clear cell renal cell carcinoma. J Clin Oncol 2007, 25:4757-4764.

32. Liao SY, Aurelio ON, Jan K, Zavada J, Stanbridge EJ: Identification of the MN/CA9 protein as a reliable diagnostic biomarker of clear cell carcinoma of the kidney. Cancer Res 1997, 57:2827-2831.

doi:10.1186/1756-9966-32-8

Cite this article as: Wang et al:: Epigenetic change in kidney tumor: downregulation of histone acetyltransferase MYST1 in human renal cell carcinoma. Journal of Experimental \& Clinical Cancer Research 2013 32:8.

\section{Submit your next manuscript to BioMed Central and take full advantage of:}

- Convenient online submission

- Thorough peer review

- No space constraints or color figure charges

- Immediate publication on acceptance

- Inclusion in PubMed, CAS, Scopus and Google Scholar

- Research which is freely available for redistribution 